\section{REVIEW ARTICLE}

R. Jain

\title{
Perfusion CT Imaging of Brain Tumors: An Overview
}

\begin{abstract}
SUMMARY: Perfusion imaging of brain tumors has been performed by using various tracer and nontracer modalities and can provide additional physiologic and hemodynamic information, which is not available with routine morphologic imaging. Tumor vascular perfusion parameters obtained by using CT or MR perfusion have been used for tumor grading, prognosis, and treatment response in addition to differentiating treatment/radiation effects and non-neoplastic lesions from neoplasms. This article is an overview of the utility of PCT for assessment of brain tumors and describes the technique, its advantages, and limitations.
\end{abstract}

\begin{abstract}
ABBREVIATIONS: $\mathrm{BBB}=$ blood-brain barrier; $\mathrm{CBF}=$ cerebral blood flow; $\mathrm{CBV}=$ cerebral blood volume; DSC = dynamic susceptibility contrast; FDG-PET = fluorodeoxyglucose-positron-emission tomography; $K^{\text {trans }}=$ volume transfer coefficient; $M R I=M R$ imaging; $M T T=$ mean transit time; $\mathrm{MVCP}=$ microvascular cellular proliferation; $\mathrm{MVD}=$ microvascular density; $\mathrm{PCT}=$ perfusion $\mathrm{CT}$; $\mathrm{PS}=$ permeability surface-area product; $\mathrm{rCBV}=$ regional cerebral blood flow; $\mathrm{ROI}=$ region of interest; rPSR = relative percentage signal recovery; SDF-1 $=$ stromal derived factor- $1 ; \mathrm{TDL}=$ tumefactive demyelinating lesion; TVA = total vascular area; VEGF = vascular endothelial growth factor; $\mathrm{WHO}=$ World Health Organization
\end{abstract}

G liomas, the most common primary brain neoplasms in adults, are very heterogeneous tumors. High-grade gliomas can be highly invasive and extremely vascular tumors. Two of the most important factors in determining the malignancy of gliomas are their ability to infiltrate the brain parenchyma and to recruit or synthesize vascular networks for further growth (ie, neoangiogenesis). ${ }^{1,2}$ Malignant brain tumors are characterized by neovascularity and increased angiogenic activity, with a higher proportion of immature and highly permeable vessels. Glioma grading is currently based on the histologic assessment of the tumor, which is achieved by either brain biopsy or cytoreductive surgery; however, there are inherent limitations with these techniques and their interpretation. ${ }^{3}$

In vivo perfusion imaging techniques provide additional information regarding tumor physiology and hemodynamics, which may help in better characterizing glioma malignancy and may also overcome some of the limitations of histologic grading and conventional morphologic imaging. Perfusion imaging has been used to assess tumor grade and prognosis and, recently, to assess treatment response, which has attracted more attention due to the advent of newer therapeutic options, including antiangiogenic agents. Traditionally, perfusion imaging of brain tumors has been performed with MR imaging, by using various perfusion imaging techniques and estimating tumor blood volume, blood flow, and permeability. ${ }^{3-5}$ However, PCT, which has also been used recently for glioma grading, ${ }^{6,7}$ provides a linear relationship between tissue attenuation and tissue concentration of a contrast agent, unlike perfusion MR imaging, and, hence, probably provides a

From the Division of Neuroradiology, Departments of Radiology and Neurosurgery, Henry Ford Health System, Detroit, Michigan.

Please address correspondence to Rajan Jain, MD, Division of Neuroradiology, Departments of Radiology and Neurosurgery, Henry Ford Health System, 2799 West Grand Blvd, Detroit MI 48202; e-mail: rajanj@rad.hfh.edu

Indicates open access to non-subscribers at www.ajnr.org

http://dx.doi.org/10.3174/ajnr.A2263 more robust and less biased estimation of hemodynamic (tumor blood volume) and physiologic (tumor vascular leakiness) parameters. In view of the wider availability, faster scanning times, and low cost compared with MR perfusion, PCT is potentially well-suited to study brain tumors ${ }^{6,7}$ and could potentially be useful as an easy tool for quantitative estimates of perfusion parameters and their use as imaging biomarkers.

\section{In Vivo Perfusion Imaging versus Histopathology}

The current standard for tumor grading is histopathologic assessment of tissue, which has inherent limitations, such as sampling error, interobserver variation, and a wide variety of classification systems that are available, the most common of which is the WHO grading system. ${ }^{3}$ Most gliomas, especially high-grade ones, show a high degree of regional heterogeneity within the tumor based on tumor cellularity, edema, and necrosis. Many of these factors are inherently dependent on the blood supply. Histologic evaluation of tumor angiogenesis by using various markers such as MVD, MVCP, and TVA is also limited by this regional heterogeneity, and its confounding effect is worsened by its small size and a limited number of samples obtained with surgical biopsy. These limitations can frequently result in inaccurate classification and grading of gliomas due to sampling errors. Hence, there is a need for noninvasive in vivo clinical imaging tools that can study perfusion in the entire tumor, can be used to assess much larger volumes than small biopsy samples, and can probably guide biopsy and excision sites for better results.

Brain tumor angiogenesis is a continuously evolving process that can also be affected by various treatment modalities. Hence, in vivo perfusion imaging that can be repeated, unlike invasive procedures such as surgical excision or biopsy, can help assess continued evolution of these tumors as well as treatment response. Another important limitation of the histologic grading system is that gliomas having similar grades respond differently to similar treatment regimens as has been noted with various molecular and genetic markers, ${ }^{8}$ suggesting that there is a role for other biomarkers such as perfusion 
parameters in predicting progression or survival, apart from histologic grading. ${ }^{3}$

\section{Perfusion Parameters and Their Importance}

Dynamic contrast-enhanced imaging techniques using MR imaging or CT have been used to obtain measures of tumor vascular physiology and hemodynamics. After the rapid administration of a contrast agent and the acquisition of serial images at short intervals (seconds), an analysis that uses a pharmacokinetic model of the time dependence of contrast can produce imaging biomarkers such as tumor blood volume, blood flow, vascular permeability, and size of extravascular extracellular space. Many of these parameters have been correlated with tumor grade, aggressiveness, and prognosis. $^{3,5,7,9}$

\section{Tumor Blood Volume}

Regional tumor blood volume measurements reflect an assessment of tumor vasculature and perfusion and have been correlated with glioma grading as well as prognosis. Measurement of tumor blood volume is a good surrogate marker for MVD, a measure of angiogenesis and an important prognostic indicator $^{10-12}$ in many human cancers. The association between MVD and tumor aggressiveness can be explained by the following: 1) Solid tumors are composed of 2 interdependent components, which include the malignant cells and the stroma that they induce, and MVD could be a measure of the success that a tumor has in forming this stromal component. 2) Endothelial cells in this stromal component stimulate the growth of tumor cells; thus, the more intratumoral vessels there are, the more endothelial cells and paracrine growth stimulation are seen. 3) Intratumoral MVD is a direct measure of the vascular window through which tumor cells pass to spread to distant sites. ${ }^{12}$ Tumoral MVD, however, does not distinguish new blood vessels from the native ones, does not mark actively proliferating endothelial cells, and does not correlate with the degree of endothelial cell proliferation. However, these limitations do not seem to diminish the clinical value of this measure. Cha et $\mathrm{al}^{13}$ showed a strong correlation of CBV measurements in mouse gliomas with MVD and suggested that $\mathrm{rCBV}$ may be elevated due to an increase in vessel size or the total number of vessels or both. Aronen et al ${ }^{14}$ also showed a strong correlation of CBV and tumor energy metabolism with MVD by using MR perfusion and FDG-PET imaging, respectively.

\section{Tumor Vascular Permeability}

Tumor blood vessels have defective and leaky endothelium. Hypoxia or hypoglycemia that occurs in rapidly growing tumors increases the expression of VEGF, which is not only a potent angiogenic factor but also a potent permeability factor. ${ }^{15,16}$ VEGF leads to the development of neoangiogenic vessels, which are immature and tortuous ${ }^{2}$ and also have increased permeability to macromolecules due to large endothelial cell gaps, incomplete basement membrane, and absence of smooth muscles. These abnormal tumor vessels can be used as potential markers to assess the tumor grade. Thus, in vivo measurement of tumor vessel permeability is important for various reasons: 1) It can be used for grading tumors because increased permeability is associated with immature blood vessels, which are seen with neoangiogenesis. 2) It can be used to study the response of tumors to various therapies, especially antiangiogenic therapy. ${ }^{17,18} 3$ ) Understanding the concept of permeability can help in understanding the mechanism of the entry of therapeutic agents into the central nervous system and 4) in the development of methods to selectively alter the $\mathrm{BBB}$ to enhance drug delivery. ${ }^{19}$

\section{PCT Technique}

PCT tracer kinetics theory has been described well in multiple previous publications. ${ }^{7,20-23}$ Perfusion studies can be performed by using multidetector row CT scanners. Currently available 16-section CT scanners can cover $2 \mathrm{~cm}$ of the brain, which is increased to $4 \mathrm{~cm}$ by using a 64-section CT scanner. A low radiation dose noncontrast CT head study is usually performed to localize the region of interest before obtaining a perfusion scan. For the perfusion scan, $50 \mathrm{~mL}$ of nonionic contrast is injected at a rate of $4-5 \mathrm{~mL} / \mathrm{s}$ through an intravenous line by using an automatic power injector. At 5 seconds into the injection, a cine (continuous) scan is initiated with the following technique: $80 \mathrm{kV}$ (peak), $100-120 \mathrm{~mA}$, and 1 second per rotation for a duration of 50 seconds. After the initial 50second cine scan, 8 more axial images can be acquired, 1 image every 15 seconds for an additional 2 minutes, thus giving a total acquisition time of 170 seconds to assess delayed permeability. ${ }^{7}$ Four 5-mm-thick axial sections are acquired with the 16-section CT scanner, whereas for a 64-section CT scanner, eight 5-mm-thick axial sections are acquired, resulting in a total coverage area of $4 \mathrm{~cm}$, instead of $2 \mathrm{~cm}$ with the 16-section scanner. Maps of perfusion parameters can be obtained by using many of the commercially available software applications. We use an Advantage Windows workstation with CT perfusion 3.0 software (GE Healthcare, Milwaukee, Wisconsin), a 2-compartment model based on adiabatic approximation of the model of John and Wilson, ${ }^{23}$ to generate CBV, CBF, MTT, and PS maps in our patients. The superior sagittal sinus is generally used as the venous output function, and the artery with the greatest peak and slope on time-attenuation curves, as the arterial input function. A region of interest is drawn within the confines of a large vessel, and the automatic function of the software picks the pixels with the greatest peak and slope on the time-attenuation curve for analysis.

\section{Relationship between Glioma Grade and PCT Parameters}

Most of the literature regarding the utility of perfusion imaging for glioma grading is based on various MR perfusion techniques. Recently, PCT has also been used to grade gliomas on the basis of perfusion parameters. ${ }^{6,7}$ Ellika et $\mathrm{al}^{6}$ were able to differentiate low- and high-grade gliomas with a high sensitivity $(85.7 \%)$ and specificity (100\%) by using PCT and a CBV normalized relative to a normal-appearing contralateral white matter threshold of 1.92. This relationship between CBV and histologic grade is intuitive because pathology studies show higher MVD in higher grade tumors. Jain et $\mathrm{al}^{7}{ }^{7}$ apart from differentiating low- and high-grade gliomas (Figs 1 and 2), could also differentiate a high-grade tumor group into grade III and grade IV on the basis of PS measurements that showed a stronger predictability compared with CBV, and they especially could differentiate enhancing grade III from grade IV on the basis of the differences in PS. This result is in keeping with 


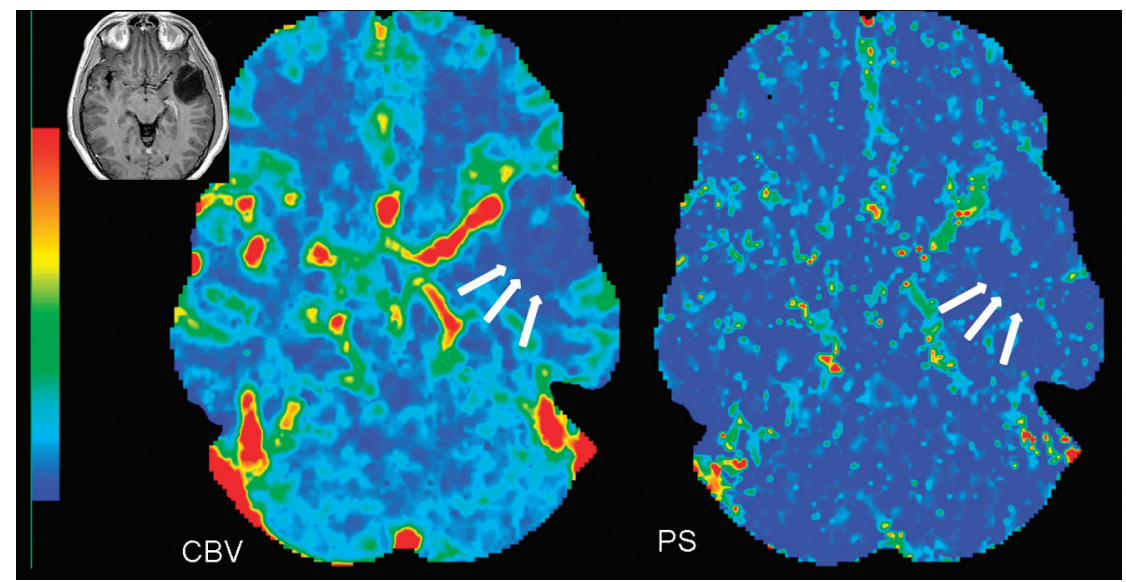

Fig 1. CBV and PS perfusion CT maps in a 45 -year-old woman with WHO grade $\|$ astrocytoma show low blood volume (CBV $=0.70 \mathrm{~mL} / 100 \mathrm{~g})$ and low permeability (PS $=0.65 \mathrm{~mL} / 100$ $\mathrm{g} / \mathrm{min}$ ) within the tumor (arrows). Inset: Postcontrast T1-weighted image shows a nonenhancing left temporal tumor with no surrounding perilesional edema.

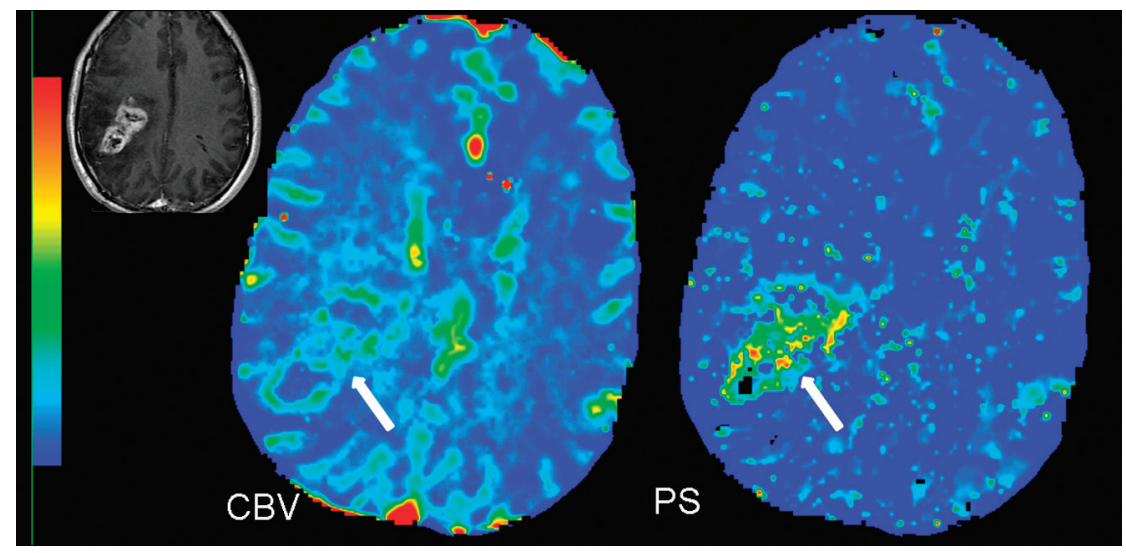

Fig 2. PCT maps in a 50-year-old man with glioblastoma multiforme. The CBV map shows high blood volume (CBV $=4.56 \mathrm{~mL} / 100 \mathrm{~g}$ ), and the PS map shows high permeability (PS $=$ $3.78 \mathrm{~mL} / 100 \mathrm{~g} / \mathrm{min}$ ) along the enhancing nodular margins of the tumor (arrow). Inset: Postcontrast T1-weighted image shows a heterogeneously enhancing mass with irregular central necrosis in the right parietal region.

the current WHO guidelines of including MVCP as a diagnostic criterion of grade IV, but not for grade III astrocytic tumors, suggesting that PS measurements could show better correlation with MVCP and, hence, could be an imaging biomarker of more immature and leaky blood vessels. Increased angiogenesis in grade IV tumors is characterized not only by an increased number of vessels compared with grade III astrocytic tumors but also by association with disproportionate lengthening, increased pliability, endothelial cell proliferation, and irregular shape, which can explain the difference in perfusion parameters for grade IV compared with grade III tumors. Nonenhancing grade III tumors have also been shown to have lower mean PS, CBV, and CBF compared with the enhancing grade III group. ${ }^{7}$ This difference is probably due to the higher MVD seen in enhancing tumors compared with that in the nonenhancing group. This could have prognostic implications because the enhancing grade III tumors show higher PS, CBV, and CBF, which may indicate more aggressive tumors with a higher recurrence rate and shorter survival periods compared with the nonenhancing grade III, which remains to be determined. Previous studies using MR perfusion have described various rCBV threshold values for glioma grading. Lev et $\mathrm{al}^{24}$ described a threshold of 1.5 in discriminating patients with low- and high-grade gliomas with a sensitiv- ity and specificity of $100 \%$ and $69 \%$, respectively. Law et al $\mathrm{l}^{25}$ showed a sensitivity and specificity of $95 \%$ and $57.5 \%$, respectively, by using 1.75 as the threshold value for $\mathrm{rCBV}$.

\section{Heterogeneity of Glioma Angiogenesis and Perfusion Imaging}

Tumor angiogenesis involves a multitude of controlled signaling cascades and structural changes that occur in a defined order and continue until a new vasculature has been formed. Tumor cellular growth usually outgrows its blood supply, leading to hypoxia, which leads to expression of hypoxia-inducible factor- $1 \alpha$ and the formation of angiogenic mediators such as VEGF and SDF-1. ${ }^{1}$ The effects of VEGF and SDF-1 lead to formation of immature and leaky blood vessels, which results in increased permeability, leading to extravasation of plasma, plasma proteins, and deposition of proangiogenic matrix proteins as well as MVCP. Later as these pericyte-poor new vessels (called "mother vessels") enlarge and give rise to "daughter vessels" through a complex series of endothelial rearrangements, MVD and TVA increase; this change, in turn, causes an increase in permeability. Finally with vessel maturation, the total number and area of blood vessels continue to increase more than the vessel leakiness, hence, evolving into a very heterogeneous tumor with various regions probably 


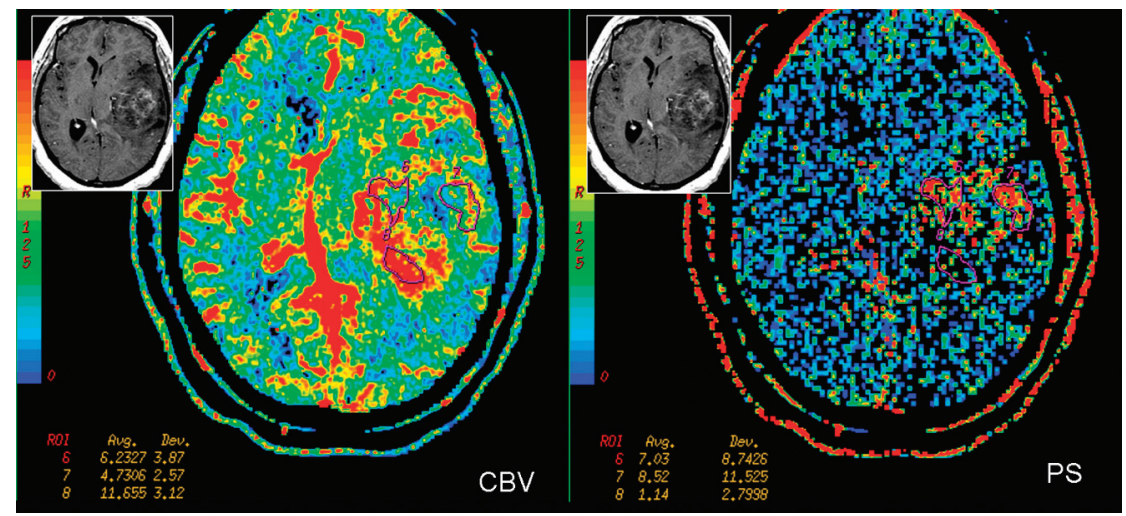

Fig 3. PCT maps in a 39-year-old woman with heterogeneously enhancing grade III glioma involving the left temporal lobe. CBV and PS maps show an enhancing region laterally. Region of interest 7 demonstrates the highest PS $(8.52 \mathrm{~mL} / 100 \mathrm{~g} / \mathrm{min})$, but not the highest CBV $(4.73 \mathrm{~mL} / 100 \mathrm{~g})$ within the tumor; whereas region of interest 8 shows relatively lower PS $(1.14$ $\mathrm{mL} / 100 \mathrm{~g} / \mathrm{min})$ but the highest CBV within the tumor $(11.65 \mathrm{~mL} / 100 \mathrm{~g})$, suggesting that there is marked heterogeneity within high-grade gliomas, which could be due to the heterogeneity of tumor angiogenesis. Inset: Postcontrast T1-weighted axial images show a heterogeneously enhancing left temporal lobe tumor.

showing different mixtures of vessel characteristics and angiogenesis, which can be seen as regions with high CBV but not very high PS and vice versa on perfusion imaging (Fig 3). Correlation of various tumor perfusion estimates with histologic angiogenesis markers could thus be very useful as far as in vivo identification of these different regions of angiogenesis is concerned and could benefit clinicians in assessing any therapeutic effects during and after administration of antitumor (or antiangiogenic) agents. MVD could indicate total tumor vasculature, including both mature and immature vessels in the tumor, whereas assessment of MVCP, pericyte-poor neovessels, and VEGF expression would indicate immature vasculature and, hence, sites of active angiogenesis. In a recent study, we have shown that CBV showed a stronger correlation with MVD $(r=0.596, P<.001)$, whereas PS showed a stronger correlation with MVCP $(r=0.546, P=.001)$, by using imageguided biopsy specimens. These findings suggest that these 2 perfusion parameters represent different aspects of tumor angiogenesis and in vivo identification, which could be of clinical importance. ${ }^{26}$ Better understanding of the histologic basis for these hemodynamic and physiologic parameters could help increase their use as imaging biomarkers.

\section{Role of PCT in Differentiating Recurrent Tumor from Radiation Necrosis}

Recent advances in brain tumor treatment have led to aggressive management strategies with combinations of surgery, chemotherapy, and radiation therapy based on the location and histologic type of tumor. In particular, various forms of radiation therapy, including stereotactic radiosurgery, highdose external beam radiation, and brachytherapy, have become important therapeutic adjuncts. Patient survival and quality of life are correlated with response to therapy, tumor recurrence, and also adverse effects of radiation therapy, such as radiation necrosis. Differentiating recurrent tumors from radiation necrosis on imaging studies has always been an important clinical imperative because the management of these 2 entities is different. The problem is confounded by the fact that there is often a mixture of tumor with necrosis. Conventional MR imaging features and MR spectroscopic imaging have been used to differentiate radiation necrosis from recurrent tumors with mixed success. ${ }^{27,28}$ Various forms of meta- bolic imaging techniques have been used in the past with limited results. FDG-PET, ${ }^{29}$ which is based on tumor glucose metabolism, has shown variable sensitivity and specificity in differentiating recurrent tumors from radiation necrosis and also has limited spatial resolution. Posttreatment recurrent enhancing lesions have also been evaluated with MR perfusion imaging, showing increased CBV in recurrent tumors compared with non-neoplastic lesions. ${ }^{30,31}$ Jain et al $^{31}$ used perfusion CT to differentiate the 2 entities, and recurrent tumors showed higher CBV and CBF, and lower MTT (Fig 4A) compared with radiation necrosis (Fig 4B). PCT could have a slight edge because most of these patients, after having undergone multiple various combination therapies have some components of hemorrhage and mineralization, which could produce susceptibility artifacts complicating the perfusion analysis, especially with the use of dynamic susceptibility-weighted imaging. ${ }^{31}$

Previous authors have successfully used relative percentage signal-intensity recovery or signal intensity enhancement-time curves as an indirect measure of vascular leakiness by using MR perfusion techniques; however, absolute quantitative estimates of permeability have not yet been used for this very important clinical scenario. Kamiryo et $\mathrm{al}^{32}$ also demonstrated that BBB architecture of capillaries within previously irradiated brain tissue remains intact despite a decrease in the mean capillary attenuation as well as increased capillary diameter. Barajas et $\mathrm{al}^{33}$ showed lower relative percentage signal recovery (rPSR) in recurrent glioblastoma multiforme compared with radiation necrosis by using DSC-MR perfusion imaging, suggesting a disrupted BBB that was more permeable to macromolecular contrast agents; however, their measurements were not a direct estimate of lesion leakiness. They also noted a large degree of overlap between the 2 groups, making rPSR a less robust predictor of recurrent tumor. The same group $^{34}$ recently demonstrated significantly lower percentage signal-intensity recovery in radiation necrosis compared with recurrent metastatic intra-axial tumors, however, also noting a major limitation of susceptibility artifacts resulting in image degradation, making DSC measurements difficult to obtain. Quantitative estimates of PS obtained by using PCT are not affected by susceptibility artifacts and can help differentiate radiation necrosis from recurrent tumors. Radiation necrosis 


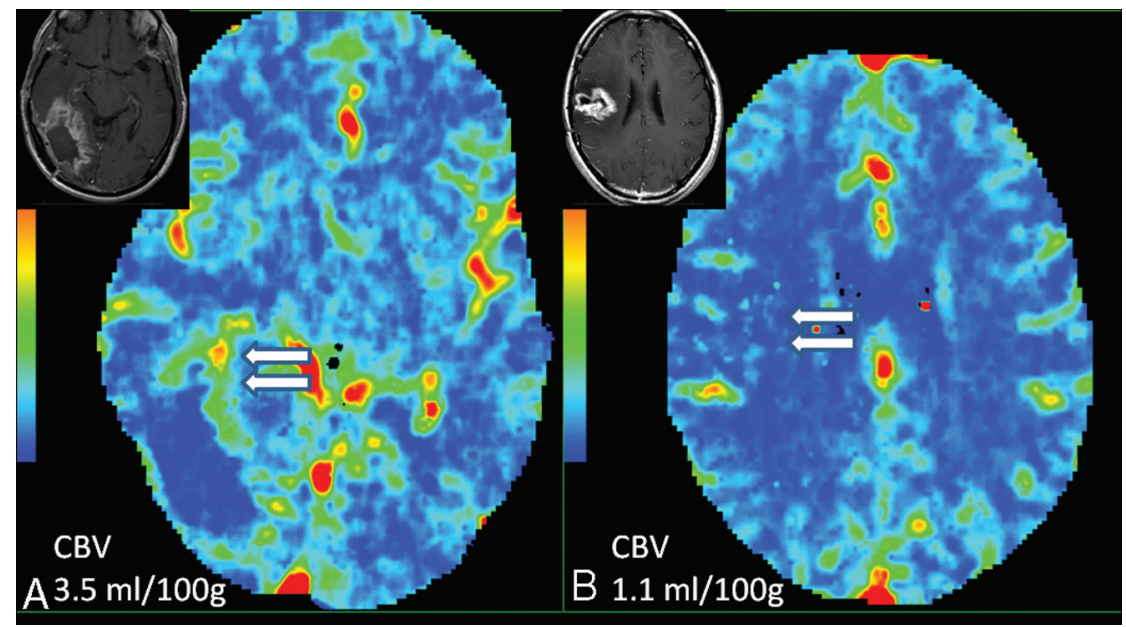

Fig 4. A, A 56-year-old man who was previously treated for a right occipital anaplastic astrocytoma, shows a progressive enhancing lesion in the medial aspect of the surgical resection cavity (inset) and PCT CBV map shows high CBV (CBV $=3.5 \mathrm{ml} / 100 \mathrm{~g}$ ), suggesting recurrent/progressive tumor (arrows). B, Histopathology proven radiation/treatment necrosis (arrows) in a 34-year-old woman who underwent radiation and chemotherapy for a right fronto-parietal anaplastic astrocytoma shows low CBV (CBV = $1.1 \mathrm{ml} / 100 \mathrm{~g}$ ) in a recurrent/progressive enhancing lesion (inset).

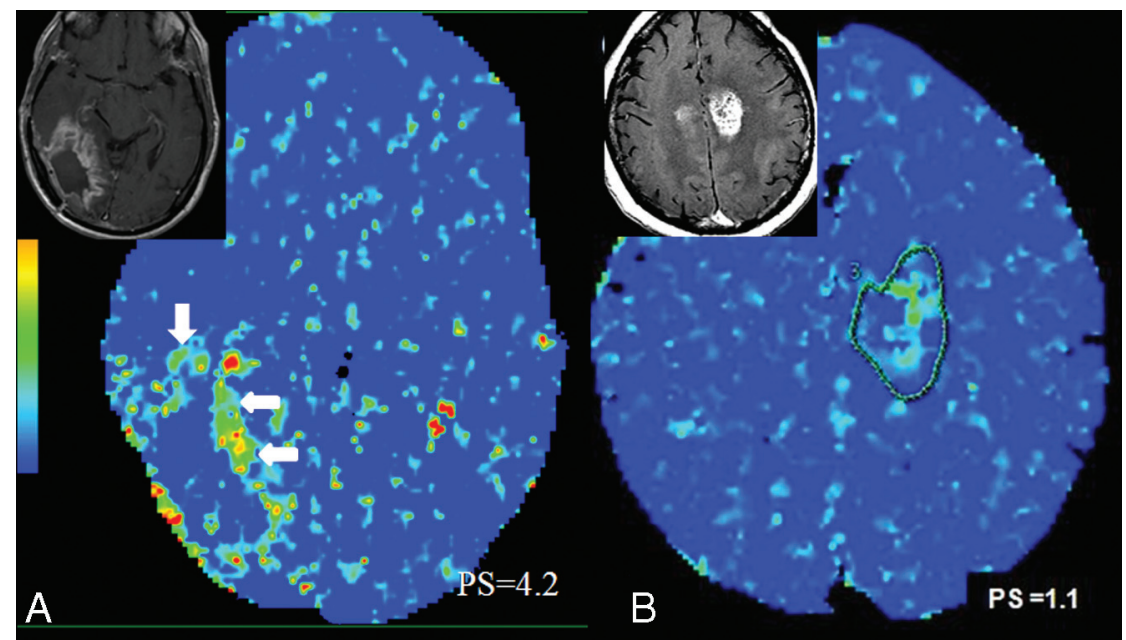

Fig 5. $A$, Same case as in Fig 4A. PCT PS map shows high PS (PS $=4.2 \mathrm{ml} / 100 \mathrm{~g} / \mathrm{min}$ ) in the medial aspect of the surgical resection cavity (arrows) in a histopathology proven recurrent/progressive tumor (inset). B, A 41-year-old man with initial diagnosis of WHO grade II astrocytoma received chemotherapy and radiation. A 33 month follow-up MR image (inset) shows development of a recurrent enhancing lesion. PCT PS map shows low PS (PS $=1.1 \mathrm{ml} / 100 \mathrm{~g} / \mathrm{min}$ ) suggesting radiation necrosis. The patient underwent open biopsy and histopathology revealed radiation necrosis without viable tumor cells.

shows lower PS in addition to lower CBV compared with recurrent tumors, probably due to the fact that recurrent tumors have much higher expression of VEGF, leading to much leakier blood vessels compared with radiation necrosis (Fig 5). ${ }^{35}$

\section{Differentiating Gliomas from Other Non-Neoplastic Lesions and from Lymphomas}

TDLs are usually solitary lesions that can mimic intracranial neoplasms on initial presentation due to their atypical morphologic MR imaging features. The situation could be confounded by the fact that TDLs can occasionally simulate neoplasms on histopathologic examination. ${ }^{36,37}$ This has mostly been attributed to limited tissue samples such as those obtained from stereotactic brain biopsy. Biopsy of the gliotic margin may be pathologically misinterpreted at the edge of a glioma, and biopsy of the center of a TDL lesion may lead to the erroneous diagnosis of an infarct or necrotic neoplasm. ${ }^{37}$ Furthermore, the numerous macrophages and reactive astro- cytes usually present in TDL biopsies may appear atypical and mimic a hypercellular lesion consistent with glioma, particularly on intraoperative smear preparations. One of the important biologic differences between TDL and high-grade intracranial tumors is the presence of neoangiogenesis and vascular endothelial proliferation in the latter, whereas TDLs are characterized by intrinsically normal or inflamed vessels without evidence of neovascularity. ${ }^{36,38,39}$ TDLs show lower PS and CBV compared with high-grade gliomas, and PCT can be used to differentiate the 2 entities (Fig 6). ${ }^{40}$

Similarly, various vasculitis and angiopathies can rarely present as solitary or multiple masslike lesions, mimicking neoplasms on morphologic imaging. PCT can be used to exclude a neoplasm by demonstrating low blood volume in these lesions (Fig 7). Lymphomas rarely can have an imaging appearance similar to that in high-grade gliomas, particularly if complicated with hemorrhage. However, lymphomas have been shown to have an intermediate increase in CBV com- 


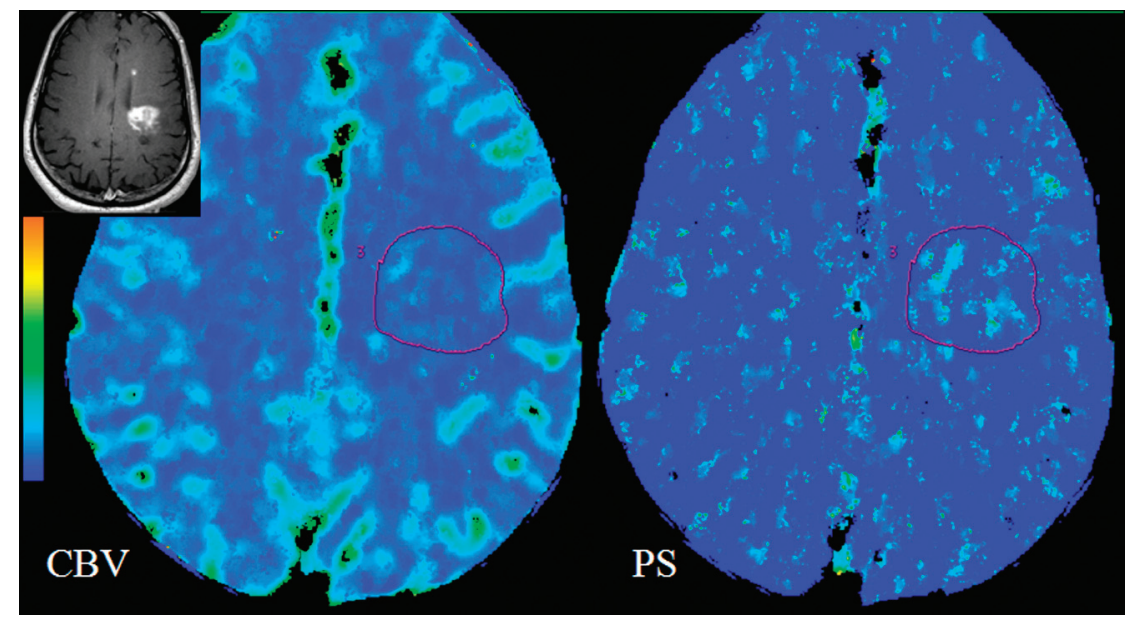

Fig 6. Histopathology-proved TDL in a 45-year-old woman who presented with a very heterogeneously enhancing large lesion in the left frontoparietal periventricular region (inset). PCT CBV and PS maps show low to minimally increased blood volume (CBV $=1.01 \mathrm{~mL} / 100 \mathrm{~g}$ ) and permeability (PS $=0.40 \mathrm{~mL} / 100 \mathrm{~g} / \mathrm{min}$ ), unlike a high-grade tumor that it was mimicking on postcontrast images, hence, suggesting a non-neoplastic lesion.

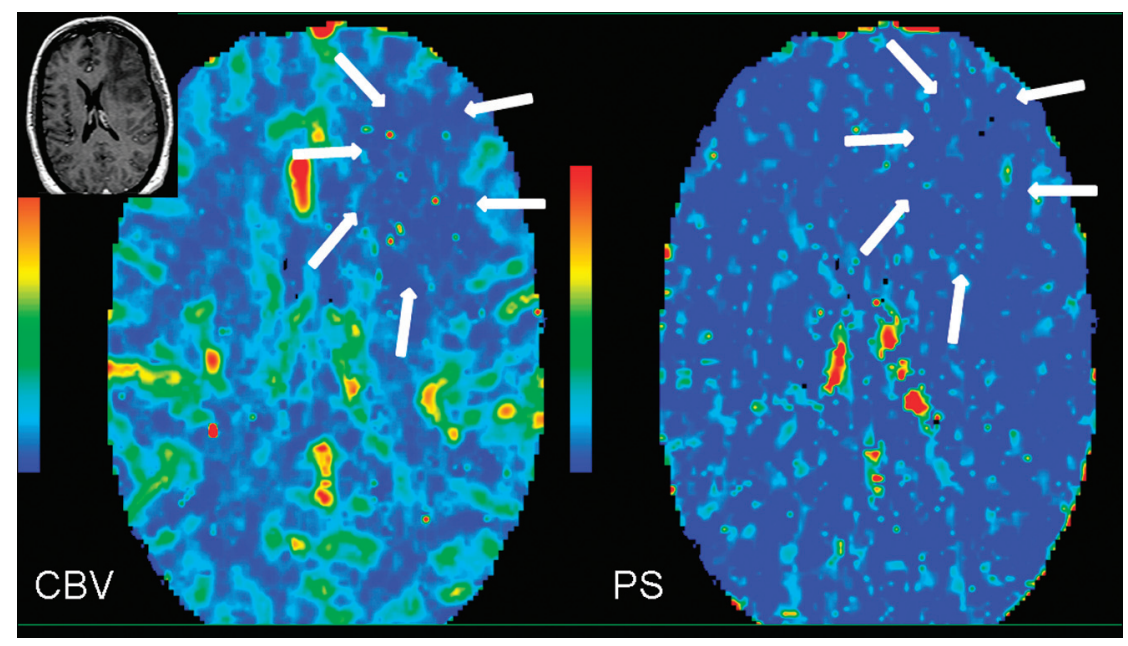

Fig 7. MR image (inset) shows a large nonenhancing mass lesion in the left frontal lobe in a 65-year-old man who presented with headaches. PCT CBV and PS maps show low blood volume and permeability (arrows), suggesting either a low-grade glioma (however, this is slightly unusual considering the associated edema and mass effect) or, more likely, a non-neoplastic lesion. The patient underwent open biopsy, which showed amyloid angiopathy.

pared with the very high CBV seen in high-grade gliomas; hence, perfusion imaging can help differentiate the 2 entities preoperatively.

\section{Advantages and Limitations of PCT for Brain Tumor Assessment}

MR perfusion techniques have certain limitations because of the nonlinear relationship of the signal intensity with the contrast, both for dynamic contrast-enhanced imaging with T1weighting $^{41}$ and for DSC imaging with T2- or T2*-weighting. In the latter case, when the contrast agent remains intravascular, the method is widely accepted as a relative estimate of CBF and $\mathrm{CBV}$, though there is a possibility for artifacts because of difficulties in assessing the shape and timing of the arterial input function. ${ }^{42}$ In the event that substantial leakage of a contrast agent from intra- to extravascular space takes place, a strong and competing $\mathrm{T} 1$ contrast effect is often noticed in the areas of pathology because of the necessity of the short $(\sim 1$ second) TR needed to estimate CBF. As a first-order tactic to minimize the competing $\mathrm{T} 1$ contrast, preloading with contrast agent has been proposed with some success. ${ }^{43}$ However, this approach does not allow an estimate of $K^{\text {trans }}$.

An alternative has also been proposed. ${ }^{44}$ To decrease the T1 effect, this approach used a slower TR, lengthening the TR of the experiment, and undermining the estimation of $\mathrm{CBF}$, thus, yielding estimates of only $\mathrm{CBV}$ and $K^{\text {trans }}$. A further refinement, allowing the estimate of blood volume and producing an index of transfer constant, has been suggested, ${ }^{43}$ and a dualecho gradient-echo sequence ${ }^{45}$ also shows some potential for an index of blood volume and transfer constant.

Despite the partial success of these rapid imaging studies, in contrast to CT perfusion, there does not appear to be an MR imaging technique that will reliably quantify $\mathrm{CBF}, \mathrm{CBV}$, and $K^{\text {trans }}$ in 1 experiment. PCT also has the advantage of providing absolute measures of these perfusion parameters, whereas MR imaging estimates are mostly relative to the normal brain parenchyma. Another major disadvantage of MR perfusion is susceptibility artifacts due to hemorrhage and various mineral depositions, which can be a major issue in posttreatment patients with tumor and also more so with dynamic susceptibil- 
ity-weighted imaging techniques. Despite these findings, MR perfusion has been used more often because MR imaging is the standard of care for patients with brain tumor, whereas PCT requires a separate examination with an iodinated contrast agent. Hence, using PCT as a follow-up tool in patients treated with various combinations of chemotherapy and radiation is not very practical.

Radiation exposure is another potential concern of PCT compared with MR perfusion techniques. However, lowradiation-dose protocols with $100-120 \mathrm{~mA}$, as presently used at our center, compared with $200 \mathrm{~mA}$, have reduced the mean effective dose $(3-4 \mathrm{mSv})$ by almost $50 \%-60 \%$ (R.J.; unpublished data) without affecting the image quality of perfusion parametric maps. In addition, some of the advanced image reconstruction techniques, such as adaptive statistical iterative reconstruction, can reduce image noise and improve low contrast detectability and image quality with $\leq 32 \%-65 \%$ reductions in CT dose index. ${ }^{46}$ Wider availability and use of these techniques could reduce the radiation dose for PCT studies quite significantly, making them more attractive for routine use. Limited coverage of the brain by using PCT has also been a limitation, particularly with 16-section CT scanners but has been improved to $4 \mathrm{~cm}$ of the brain by using 64-section CT scanners and, hence, can cover most tumors.

\section{Conclusions}

Clinically available perfusion imaging tools whether by using MR imaging or CT can provide additional information regarding brain tumor vascular estimates, which could be useful imaging biomarkers for preoperative glioma grading and angiogenesis assessment and could also be useful for treatment planning and response assessment. PCT has the advantage of providing 2 of the most important tumor vascular estimates (ie, blood volume and permeability) in 1 single experiment as well as providing a linear relationship of tissue signal intensity with tissue contrast agent and the availability of an arterial input function unlike that in most of the available MR perfusion techniques; hence, PCT has the potential to be a useful tool for brain tumor assessment.

\section{References}

1. Folkman J. The role of angiogenesis in tumor growth. Semin Cancer Biol 1992;3:65-71

2. Jain RK, Munn LL, Fukumura D. Dissecting tumour pathophysiology using intravital microscopy. Nat Rev Cancer 2002;2:266-76

3. Law M, Young RJ, Babb JS, et al. Gliomas: predicting time to progression or survival with cerebral blood volume measurements at dynamic susceptibility-weighted contrast-enhanced perfusion MR imaging. Radiology 2008;247:490-98

4. Roberts HC, Roberts TP, Brasch RC, et al. Quantitative measurement of microvascular permeability in human brain tumors achieved using dynamic contrast-enhanced MR imaging: correlation with histologic grade. AJNR Am J Neuroradiol 2000;21:891-99

5. Law M, Yang S, Babb JS, et al. Comparison of cerebral blood volume and vascular permeability from dynamic susceptibility contrast-enhanced perfusion MR imaging with glioma grade. AJNR Am J Neuroradiol 2004;25:746-55

6. Ellika SK, Jain R, Patel SC, et al. Role of perfusion CT in glioma grading and comparison with conventional MR imaging features. AJNR Am J Neuroradiol 2007;28:1981-87

7. Jain R, Ellika SK, Scarpace L, et al. Quantitative estimation of permeability surface-area product in astroglial brain tumors using perfusion $\mathrm{CT}$ and correlation with histopathologic grade. AJNR Am J Neuroradiol 2008;29:694-700

8. Cairncross JG, Ueki K, Zlatescu MC, et al. Specific genetic predictors of che- motherapeutic response and survival in patients with anaplastic oligodendrogliomas. J Natl Cancer Inst 1998;90:1473-79

9. Law M, Oh S, Johnson G, et al. Perfusion magnetic resonance imaging predicts patient outcome as an adjunct to histopathology: a second reference standard in the surgical and nonsurgical treatment of low-grade gliomas. Neurosurgery 2006;58:1099-107

10. Leon SP, Folkerth RD, Black PM. Microvessel density is a prognostic indicator for patients with astroglial brain tumors. Cancer 1996;77:362-72

11. Li VW, Folkerth RD, Watanabe $\mathrm{H}$, et al. Microvessel count and cerebrospinal fluid basic fibroblast growth factor in children with brain tumours. Lancet 1994:344:82-86

12. Weidner N. Intratumor microvessel density as a prognostic factor in cancer. Am J Pathol 1995;147:9-19

13. Cha S, Johnson G, Wadghiri YZ, et al. Dynamic, contrast-enhanced perfusion MRI in mouse gliomas: correlation with histopathology. Magn Reson Med 2003;49:848-55

14. Aronen HJ, Pardo FS, Kennedy DN, et al. High microvascular blood volume is associated with high glucose uptake and tumor angiogenesis in human gliomas. Clin Cancer Res 2000;6:2189-200

15. Plate $\mathrm{KH}$, Breier G, Weich HA, et al. Vascular endothelial growth factor is a potential tumour angiogenesis factor in human gliomas in vivo. Nature 1992;359:845-48

16. Shweiki D, Itin A, Soffer D, et al. Vascular endothelial growth factor induced by hypoxia may mediate hypoxia-initiated angiogenesis. Nature 1992;359:843-45

17. Bhujwalla ZM, Artemov D, Natarajan K, et al. Reduction of vascular and permeable regions in solid tumors detected by macromolecular contrast magnetic resonance imaging after treatment with antiangiogenic agent TNP-470. Clin Cancer Res 2003;9:355-62

18. Raatschen HJ, Simon GH, Fu Y, et al. Vascular permeability during antiangiogenesis treatment: MR imaging assay results as biomarker for subsequent tumor growth in rats. Radiology 2008;247:391-99

19. Provenzale JM, Mukundan S, Dewhirst M. The role of blood-brain barrier permeability in brain tumor imaging and therapeutics. AJR Am J Roentgenol 2005; 185:763-67

20. Johnson JA, Wilson TA. A model for capillary exchange. Am J Physiol 1966;210:1299-303

21. Purdie TG, Henderson E, Lee TY. Functional CT imaging of angiogenesis in rabbit VX2 soft-tissue tumour. Phys Med Biol 2001;46:3161-75

22. Lee TY, Purdie TG, Stewart E. CT imaging of angiogenesis. Q J Nucl Med 2003;47:171-87

23. St Lawrence KS, Lee TY. An adiabatic approximation to the tissue homogeneity model for water exchange in the brain: I. Theoretical derivation. J Cereb Blood Flow Metab 1998;18:1365-77

24. Lev MH, Ozsunar Y, Henson JW, et al. Glial tumor grading and outcome prediction using dynamic spin-echo MR susceptibility mapping compared with conventional contrast-enhanced MR: confounding effect of elevated rCBV of oligodendrogliomas [corrected]. AJNR Am J Neuroradiol 2004;25:214-21

25. Law M, Yang S, Wang $H$, et al. Glioma grading: sensitivity, specificity, and predictive values of perfusion MR imaging and proton MR spectroscopic imaging compared with conventional MR imaging. AJNR Am J Neuroradiol 2003;24:1989-98

26. Jain R, Gutierrez J, Narang J, et al. In vivo correlation of tumor blood volume and permeability with histological and molecular angiogenic markers in gliomas. AJNR Am J Neuroradiol. 2011;32:388-94

27. Kumar AJ, Leeds NE, Fuller GN, et al. Malignant gliomas: MR imaging spectrum of radiation therapy- and chemotherapy-induced necrosis of the brain after treatment. Radiology 2000;217:377-84

28. Chernov M, Hayashi M, Izawa M, et al. Differentiation of the radiation-induced necrosis and tumor recurrence after gamma knife radiosurgery for brain metastases: importance of multi-voxel proton MRS. Minim Invasive Neurosurg 2005;48:228-34

29. Langleben DD, Segall GM. PET in differentiation of recurrent brain tumor from radiation injury. J Nucl Med 2000;41:1861-67

30. Covarrubias DJ, Rosen BR, Lev MH. Dynamic magnetic resonance perfusion imaging of brain tumors. Oncologist 2004;9:528-37

31. Jain R, Scarpace L, Ellika S, et al. First-pass perfusion computed tomography: initial experience in differentiating recurrent brain tumors from radiation effects and radiation necrosis. Neurosurgery 2007;61:778-86, discussion $786-87$

32. Kamiryo T, Lopes MB, Kassell NF, et al. Radiosurgery-induced microvascular alterations precede necrosis of the brain neuropil. Neurosurgery 2001;49:40914, discussion 414-15

33. Barajas RF Jr, Chang JS, Segal MR, et al. Differentiation of recurrent glioblastoma multiforme from radiation necrosis after external beam radiation therapy with dynamic susceptibility-weighted contrast-enhanced perfusion MR imaging. Radiology 2009;253:486-96

34. Barajas RF, Chang JS, Sneed PK, et al. Distinguishing recurrent intra-axial metastatic tumor from radiation necrosis following gamma knife radiosurgery using dynamic susceptibility-weighted contrast-enhanced perfusion MR imaging. AJNR Am J Neuroradiol 2009;30:367-72 
35. Jain R, Narang J, Schultz L, et al. Permeability estimates in histopathology proven treatment induced necrosis using perfusion CT: can these add to other perfusion parameters in differentiating from recurrent/progressive tumors? AJNR Am J Neuroradiol. 2011;32:658-63

36. Sugita $Y$, Terasaki M, Shigemori M, et al. Acute focal demyelinating disease simulating brain tumors: histopathologic guidelines for an accurate diagnosis. Neuropathology 2001;21:25-31

37. Annesley-Williams D, Farrell MA, Staunton H, et al. Acute demyelination, neuropathological diagnosis, and clinical evolution. J Neuropathol Exp Neurol 2000;59:477-89

38. Zagzag D, Miller DC, Kleinman GM, et al. Demyelinating disease versus tumor in surgical neuropathology: clues to a correct pathological diagnosis. Am J Surg Pathol 1993;17:537-45

39. Prineas JW, MacDonald WI. Demyelinating diseases. In: Graham DI, Lantos PL, eds. Greenfield's Neuropathology. 6th ed. London, UK: Oxford University Press; 1997:814-46

40. Jain R, Ellika S, Lehman NL, et al. Can permeability measurements add to blood volume measurements in differentiating tumefactive demyelinating lesions from high grade gliomas using perfusion CT? J Neurooncol 2010;97:383-88
41. Yankeelov TE, Rooney WD, Huang W, et al. Evidence for shutter-speed variation in CR bolus-tracking studies of human pathology. NMR Biomed 2005; $18: 173-85$

42. Conturo TE, Akbudak E, Kotys MS, et al. Arterial input functions for dynamic susceptibility contrast MRI: requirements and signal options. J Magn Reson Imaging 2005;22:697-703

43. Boxerman JL, Schmainda KM, Weisskoff RM. Relative cerebral blood volume maps corrected for contrast agent extravasation significantly correlate with glioma tumor grade, whereas uncorrected maps do not. AJNR Am J Neuroradiol 2006;27:859-67

44. Johnson G, Wetzel SG, Cha S, et al. Measuring blood volume and vascular transfer constant from dynamic, $\mathrm{T}(2)^{\star}$-weighted contrast-enhanced MRI. Magn Reson Med 2004;51:961-68

45. Uematsu H, Maeda M. Double-echo perfusion-weighted MR imaging: basic concepts and application in brain tumors for the assessment of tumor blood volume and vascular permeability. Eur Radiol 2006;16:180 -86

46. Hara AK, Paden RG, Silva AC, et al. Iterative reconstruction technique for reducing body radiation dose at CT: feasibility study. AJR Am J Roentgenol 2009;193:764-71 